This item was submitted to Loughborough's Research Repository by the author.

Items in Figshare are protected by copyright, with all rights reserved, unless otherwise indicated.

\title{
The influence of model parameters on model validation
}

PLEASE CITE THE PUBLISHED VERSION

https://doi.org/10.1080/10255842.2019.1614173

PUBLISHER

(c) Taylor \& Francis

VERSION

AM (Accepted Manuscript)

PUBLISHER STATEMENT

This is an Accepted Manuscript of an article published by Taylor \& Francis in Computer Methods in Biomechanics and Biomedical Engineering on 20 May 2019, available online:

http://www.tandfonline.com/10.1080/10255842.2019.1614173.

\section{LICENCE}

CC BY-NC-ND 4.0

\section{REPOSITORY RECORD}

Infantolino, Benjamin W., Steph Forrester, Matthew Pain, and John H. Challis. 2019. "The Influence of Model Parameters on Model Validation". Loughborough University. https://hdl.handle.net/2134/38074. 
TITLE: $\quad$ The influence of model parameters on model validation.

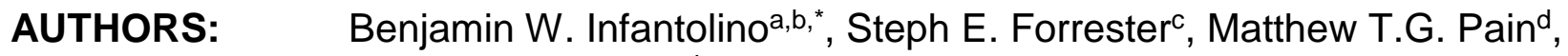
and John $\mathrm{H}$. Challis ${ }^{\mathrm{b}}$

( ${ }^{\star}$ Author to whom all correspondence should be addressed)

\section{AFFILIATIONS:}

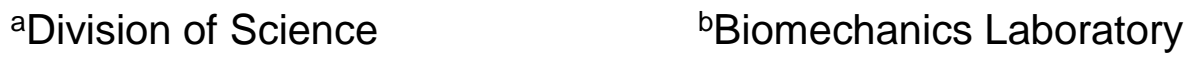

Pennsylvania State University Pennsylvania State University

Berks Campus

USA

University Park

USA

cWolfson School

Loughborough University

dSchool of Sport, Exercise \& Health Sciences

Loughborough

Leics LE11 3TU

Loughborough University

Loughborough

Leics LE11 3TU

UK

UK

\section{ADDRESS FOR CORRESPONDENCE:}

Pennsylvania State University

Berks Campus

PO Box 7009

Tulpehocken Road

PA 19610

USA

Tel \#. $\quad+(610)-396-6153$

FAX \#: $\quad+(610)-396-6155$

E-mail: bwi100@psu.edu

WORD COUNT: $\quad$ Abstract $-99(\leq 100)$, Main body - $3999(<4000)$

SUBMITTED: $\quad$ Computer Methods in Biomechanics and Biomedical Engineering (Revision Submitted - February 27, 2019)

(Submitted - July 1, 2018) 


\begin{abstract}
The study examined the sensitivity of two musculoskeletal models to the parameters describing each model. Two different models were examined: a phenomenological model of human jumping with parameters based on live subject data, and the second a model of the First Dorsal Interosseous with parameters based on cadaveric measurements. Both models were sensitive to the model parameters, with the use of mean group data not producing model outputs reflective of either the performance of any group member or the mean group performance. These results highlight the value of subject specific model parameters, and the problems associated with model validation.
\end{abstract}

KEYWORDS (3-6 words): muscle, simulation, modeling 


\section{INTRODUCTION}

Models of the human musculoskeletal system are being used to provide insights into various aspects of human movement for example, to examine healthy gait (Anderson and Pandy, 2001), gait for patients with cerebral palsy (Fox et al., 2009), jumping (van Soest et al., 1993), and cycling (Neptune et al., 2000). These models can be considered to have three components: a model component representing the muscles, a model component representing system mechanics, and a model component representing the control of the system. With any model its performance is dependent on appropriate model formulation and having appropriate parameters for the various model components.

It is imperative that models should be verified and validated so that there is confidence in the model results. Verification refers to determining that the model components are analysed (solved) correctly (Schwer, 2002); so the mathematical model is accurately represented and then solved appropriately (Fetzer, 1988). Of course a model can be verified but not provide meaningful output if the model is not appropriate. To ensure a model is appropriate the model is validated, which typically means the model output is compared with some criterion data. A model not only comprises the equations which describe the components of the model but also the model parameters. In biomechanics determining model parameters is often a challenge because it is hard to measure the model specific parameters in vivo.

Although muscle models have proven a powerful tool for the analysis of human movement, there is, unfortunately, a dearth of data on which to parameterize these models. If models of the lower limb (e.g., Delp et al., 1990; Dorn et al., 2012) are used as an example the most common sources of data are muscle parameters from the studies of Wickiewicz et al. (1983) and Friederich and Brand (1990), yet these two studies dissected only three and two cadavers respectively. In fact, neither study provides sufficient information to fully parameterize a muscle model so data from the two studies are often combined (e.g., Hoy et al., 1990). There are some other studies which are more comprehensive in nature, for example Ward et al. (2009) provided a set 
of average parameters for the major muscles of the lower limb from the detailed dissection of 21 limbs. Even with some larger parameter sets becoming available some type of scaling is required to model specific subjects (e.g., Zajac, 1989), which is not necessarily straightforward as the variability of muscle model parameters can be greater than the variability of basic human anthropometry (Infantolino and Challis, 2014). A methodological review by Valero-Cuevas et al. (2009) discussed a range of considerations for neuromuscular modelling which included the difficulty of determining model parameters, and highlighted the importance of assessing the sensitivity of the model to input parameters. They proposed an approach, Monte Carlo evaluations, to deal with insufficient knowledge of model parameters. However, even in situations where mean input data can be obtained with a high degree of confidence, Cook and Robertson (2017) demonstrated, using two biomechanical models and synthetic input data, that the resulting model output does not equate to the mean output from the individual models. This results from the non-linearity of the biomechanical models and raises the further question of the appropriateness of using mean input parameters in order to generate a mean output as a representation of a population mean response.

Given the value of musculoskeletal models but the corresponding lack of large databases of muscle model parameters it is of interest to examine how model performance depends on model parameters. Therefore, the purpose of this study was to examine the sensitivity of two different musculoskeletal models to the parameters describing the model. Specifically, the influence on model validity of using model parameters based on group mean data was investigated. These comparisons can be used to provide an estimate of the error associated with relying on mean data for model parameters as opposed to subject specific values in computer simulation modelling. Two models were examined; one was a phenomenological model of human jumping comprising a simple lumped-parameter model of jumping based on live subject data, and the other a model of the First Dorsal Interosseous causing abduction of the second metacarpalphalangeal joint with model parameters based on cadaveric measurements. These two models reflect different approaches to determining model parameters. In the model of jumping experimental data from experimental subjects is used to determine 
model parameters, such an approach has been used in many studies (e.g., Allen et al., 2011; Forrester et al., 2011). The second model was of the First Dorsal Interosseous muscle and reflects the approach that muscle morphological data is used specify the muscle model parameters (e.g., Delp et al., 1990; Jacobs et al., 1990). These two models analyses will be presented sequentially before a common Discussion section.

\section{MODEL 1 - HUMAN JUMPING}

Overview: A simple two-segment moment-driven jumping model (Alexander, 1990) was used to examine the effects of model input parameters on maximal drop jumping ability by performing two different types of 'average parameter' modelling. This model format has been adopted by others for the analysis of jumping (e.g., Challis, 1998; Cheng and Hubbard, 2004 Seyfarth et al., 2000). Firstly, a population of 15 subject specific models were created based on anthropometrics and knee extensor strength data from 15 subjects. These subject specific models were then utilised by examining, in effect, a perfect modelling situation where subject specific simple models of jumping were the population. In addition simulations were run based on mean subject data. The simulations based on mean data were compared to the individual and group average subject specific model results. The same model determination procedure, simulation protocols and code were used for all test data.

$<<<$ Insert Figure 1 Around Here. $>>>$

Subject Specific Data Collection: Fifteen males volunteered for the study (age $25.5 \pm$ 5.3 years; body mass $76.3 \pm 10.9 \mathrm{~kg}$; height $1.78 \pm 0.05 \mathrm{~m}$; Table 1). Each subject gave their informed consent, and the study was conducted in accordance with the approval given by the Loughborough University Ethical Advisory Committee. $<<<$ Insert Table 1 around here. $>>>$

Maximal effort knee extensions were performed on a Cybex NORM dynamometer (CSMI, Stoughton, MA, USA). The protocol comprised of maximal isometric muscle actions at crank arm angles of $15^{\circ}, 30^{\circ}, 45^{\circ}, 60^{\circ}$ and $75^{\circ}$ (where $0^{\circ}$ represents full 
extension), followed by maximal concentric-eccentric muscle actions at preset crank arm velocities of $\pm 50^{\circ} \mathrm{s}^{-1}, \pm 100^{\circ} \mathrm{s}^{-1}, \pm 150^{\circ} \mathrm{s}^{-1}, \pm 200^{\circ} \mathrm{s}^{-1}, \pm 250^{\circ} \mathrm{s}^{-1}$ and $\pm 300^{\circ} \mathrm{s}^{-1}$, employing the pre-activation technique of Yeadon et al. (2006).

Model of Jumping: In brief, the model comprised two massless segments representing the thighs and shanks, while the body mass is represented by a point mass at the hip joint (Figure 1). There was no foot, and ground contact was represented by a pivot point at the distal end of the shank segment. The model was driven by a single knee extensor and the ground reaction forces were assumed to act through the hip. The knee extensor includes a contractile component and a series elastic component. The model was applied to a two-legged jump initiated after a drop from a height of $0.40 \mathrm{~m}$. The goal was to activate the model knee extensor such that the reactivity coefficient, defined as jump height divided by ground contact time (Cronin and Hansen, 2005), was maximised.

Extensor Model: The knee extensors were modelled using a seven parameter moment-angular velocity maximal strength curve (Yeadon et al., 2006; Forrester et al., 2011). This model combined a Hill-type moment-angular velocity curve, with a differential activation curve to account for the inhibition commonly observed during maximal voluntary eccentric and low velocity concentric muscle actions (Westing et al., 1990; Pain et al., 2013). For the concentric phase the moment $(T)$ at a given angular velocity $(\omega)$ was modelled using a hyperbola (Hill, 1938),

$$
T(\omega)=\frac{C}{\left(\omega_{C}+\omega\right)}-T_{C}
$$

Where,

$$
\begin{aligned}
& \omega_{C} \text { - constant } \\
& C=T_{C}\left(\omega_{M A X}+\omega_{C}\right) \\
& T_{C}=\frac{T_{O} \omega_{C}}{\omega_{M A X}} \\
& \omega_{M A X} \text { - maximum concentric angular velocity } \\
& T_{O} \text { - maximum isometric moment (per leg) }
\end{aligned}
$$


The eccentric phase moment was also modelled using a hyperbola,

$$
T(\omega)=\frac{E}{\left(\omega_{E}-\omega\right)}+T_{M A X}
$$

Where,

$$
\begin{aligned}
& \omega_{E}=\frac{\left(T_{M A X}-T_{O}\right)}{k T_{O}} \frac{\omega_{M A X} \omega_{C}}{\left(\omega_{\text {MAX }}+\omega_{C}\right)} \\
& k-\text { constant } \\
& E=-\left(T_{M A X}-T_{O}\right) \omega_{E} \\
& T_{M A X}-\text { maximum moment produced under eccentric conditions (per leg) }
\end{aligned}
$$

The differential activation function was represented by a sigmoid ramp function,

$$
a(\omega)=a_{\min }+\frac{\left(a_{\max }-a_{\min }\right)}{\left[1+\exp \left(\frac{-\left(\omega-\omega_{1}\right)}{\omega_{r}}\right)\right]}
$$

Where,

$$
\begin{aligned}
& a_{\min } \text { - lowest level of activation in eccentric phase } \\
& a_{\max } \text { - highest level of activation in eccentric phase } \\
& \omega_{1} \text { - the angular velocity at the midpoint in activation rise } \\
& \omega_{r} \text { - governs the rate at which activation increases with angular velocity }
\end{aligned}
$$

Overall this gives seven model parameters, four for the moment-angular velocity model $\left(T_{\text {MAX }}, T_{O}, \omega_{\text {MAX }}, \omega_{C}\right)$, and three for the differential activation curve $\left(a_{\min }, \omega_{1}, \omega_{r}\right)$. The model parameters were all determined for each subject by fitting the model to experimental data obtained from an isovelocity dynamometer using the Simulated Annealing algorithm (Corana et al., 1987, Table 2). Multiple initial estimates and random generation of potential solutions at each iterations help ensure that the parameter set determined by the Simulated Annealing algorithm represent a unique solution. 
Tendon stiffness $\left(k_{t}\right)$ was estimated using literature data (Pierrynowski, 1995; Jacobs et al., 1996) fitted to the individual subjects of this study, and assuming a maximum tendon stretch of six percent (Karamanidis and Arampatzis, 2006) (Table 2).

$$
k_{t}=\sum_{R F, V I, V L, V M} \frac{F_{O}^{\max } d_{M}}{0.06 L_{T S L}}
$$

Where,

$F_{O}{ }^{\max }$ - maximum isometric force

$d_{M}$ - moment arm

$L_{T S L}$ - tendon slack length

$<<<$ Insert Table 2 around here. $>>>$

Simulations: The initial conditions for the jump simulations were zero horizontal

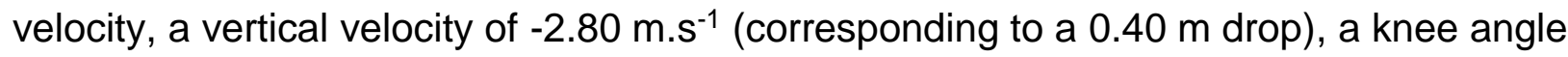
of 170 degrees, and a center of mass angle of 90 degrees. Knee extensor moment was determined as the product of the maximal knee extensor strength curve and activation level.

$$
T_{\text {OUT }}(\omega, t)=T(\omega) \times \operatorname{act}(t)
$$

The activation (act) of the knee extensor model was selected to maximize the reactivity coefficient $(\mathrm{RC})$. The activation dynamics were described by the four parameter ramp function $\left(a_{O}, a_{F}, t_{O}\right.$, and $\left.t_{R}\right)$,

$$
\operatorname{act}(t)=a_{O}+\frac{\left(a_{F}-a_{O}\right)}{\left[1+\exp \left(\frac{-\left(t-t_{O}\right)}{t_{R}}\right)\right]}
$$

Where,

$a_{O}$ - initial activation level

$a_{F}$ - final activation level

$t_{R}$ - governs the rate at which activation increases with time

$t_{O}$ - the time at the midpoint in activation rise 
Which gave an actual rise time from $a_{O}$ to $a_{F}$ of approximately $9.2 t_{R}$. The initial activation was set to zero, i.e., $a_{O}=0$, and the final activation was assumed to be maximal, i.e. $a_{F}=1$, leaving just two parameters to optimise $\left(t_{0}\right.$ and $\left.t_{R}\right)$ which were determined using the Simulated Annealing algorithm (Corana et al., 1987).

Simulations were performed using subject specific model parameters. In addition simulations were performed for two mean data sets. The first mean data set, Mean 1, was the mean of subject specific model parameters. The second mean data set, Mean 2 , reflected the more typical modelling scenario where the mean measured human strength and anthropometric data were used as the inputs to create a single 'average model'. The following model outputs were compared between model parameters sets: the reactivity coefficient, jump height, and ground contact time.

Results: There was a relatively wide range in anthropometrics and model parameters of the subjects (Tables 1 and 2) and also a wide range of reactivity coefficients, jump heights, and ground contact times for each subject specific model (Table 3). As such the standard deviation (SD) compared favorably with empirical jumping studies, for example the coefficient of variation in jump height was $\pm 17 \%$ compared with experimental values of $\pm 16 \%$ and $\pm 19 \%$ in McErlain-Naylor et al. (2014) and Pain (2014) respectively. The performance results for the mean model parameter set calculated from the 15 subject specific models, Mean 1, were within half a SD of the group mean, whereas the model using the subjects' raw data, Mean 2, had results that were at least one SD away.

$<<<$ Insert Table 3 around here. $>>>$

\section{MODEL 2 - FIRST DORSAL INTEROSSEOUS MUSCLE MODEL}

Overview: A muscle model was investigated using architectural parameters measured from eight First Dorsal Interosseous (FDI) muscles. The parameters measured on cadavers were: optimum fiber length, tendon slack length, physiological cross-sectional area, pennation angle, and muscle moment arm. For simulated static conditions the 
cadaver-specific results were compared against different representations of the group data. The FDI was selected for this study because in humans it is a rare instance of only one muscle being responsible for a particular joint motion, it abducts the index finger about the metacarpophalangeal joint (Masquelet et al., 1986).

Muscle Model: The muscle model used here was a Hill-type model similar in formulation to other models presented in the literature (e.g., Zajac, 1989; Slager et al., 1997; Lichtwark and Wilson, 2008; Miller and Hamill, 2009). The model consisted of a contractile element, and a series elastic element (Figure 2). The contractile element had force-length properties. It was assumed that the tendon had a linear stress-strain curve (Arampatzis et al., 2005). Maximum isometric muscle actions were simulated so the active state of the model was assumed to be 1 ( $q=1$, see equation in Figure 2), and the force-velocity properties of the muscle fibers could be ignored.

$$
<<<\text { Insert Figure } 2 \text { around here. }>>>
$$

Under the isometric conditions examined the muscle forces were determined using the following procedure. From the cadaver data for a given joint angle the length of the muscle-tendon complex was known. Initially muscle fiber length was computed assuming the tendon was at its slack length and the fibers comprised the remainder of the length of the muscle-tendon complex length. Muscle force was computed for this length. Given this estimate of muscle force, the tendon stretch was computed and muscle fiber length recomputed. The muscle fiber length was computed for this revised fiber length, and once again muscle force and tendon length computed. This procedure was repeated iteratively until no changes in fiber or tendon lengths were recorded.

Muscle Model Parameters: The necessary muscle model parameters were obtained from the dissection of eight cadaver hands. These data represent a sub-set of the data presented in Infantolino and Challis (2010). In the following the methods for data collection will be outlined, for more detail consult Infantolino and Challis (2010).

For each of the eight hands the moment arm of these FDI was estimated using the tendon-excursion method (An et al., 1983). The moment arm was determined for the 
typical in vivo range of motion for the abduction of the second metacarpalphalangeal joint of $20^{\circ}$ (Kendall et al., 1993). Angles were defined so that $0^{\circ}$ of abduction described the position in which the second distal phalanx was in-line with the second metacarpal (Figure 2). The cadaver hands were placed in a specially designed rig which fixed all skeletal components while the second metacarpalphalangeal joint was moved through a physiologic range of motion. During this motion both tendon excursion (via a linear cable extensometer), and joint angle (via a goniometer) were recorded. Varying order polynomials were fit to the joint angle tendon excursion data, for each cadaver's data polynomials of higher than a first order polynomial had residuals to the fit which were smaller than the noise level in the measurements, therefore the first order polynomials were used for subsequent analysis. As first derivative order polynomials were used the moment arm of the muscles were constant for the joint range of motion studied.

For the FDls from each of the cadaver hands pennation angles were measured in both heads using a standard goniometer. As the length of the fascicles varied between samples all pennation angles were referenced to optimum fiber length, this was achieved using a planimetric model of muscle geometry (Otten, 1988). In the planar muscle model muscle area remained constant, mirroring the constant volume requirement in muscle (Baskin and Paolini, 1966); to achieve this constraint muscle thickness was assumed to remain constant irrespective of fascicle length and therefore other aspects of muscle geometry could be computed (Figure 2). The mass of each muscle was measured to the nearest $0.01 \mathrm{~g}$ immediately after dissection, and then used in combination with fiber length and pennation angle to compute muscle physiological cross-sectional area (PCSA; Narici, 1999). External tendon length $\left(L_{T}\right)$ and muscle belly length were measured to the nearest $0.5 \mathrm{~mm}$ using a standard rule and a stereo dissecting microscope at $5 x$ magnification.

From each FDI two muscle fascicles were removed from each head of the FDI and placed in $20 \%$ nitric acid to digest the connective tissue surrounding the muscle fibers (Close, 1964). Following acid digestion forceps were used to remove individual fibers portions of approximately $5 \mathrm{~mm}$ in length. Digital images of the fiber sections were 
taken from a light microscope and in these the number of sarcomeres counted. Given the length of the muscle belly, the length of these samples, the number of sarcomeres in each sample, and the optimal sarcomere length for human muscle fibers $(2.7 \mu \mathrm{m}$; Walker and Schrodt, 1974) the optimum muscle belly length was computed. Optimal muscle belly length was computed since muscle fascicles were much shorter than the muscle belly and the entire muscle belly was acting on the tendon.

Other Model Parameters: There are model parameters which were not determined from the dissection of the cadavers. Many muscle models treat these model parameters as generic, that is they are general across all muscles (e.g., Zajac, 1989). The specification of the following will be outlined: width of the force-length curve, tendon strain under maximum isometric force, and the maximum isometric force.

Isolated muscle fibers can exert force for a length approximately $50 \%$ shorter than the optimum length and $50 \%$ longer that that length (Gordon et al., 1966). But the spread of force-length curve is influenced by variation in optimum fiber lengths of the fibers comprising a muscle (Huijing, 1985). To account for this variation the parameter in the force-length equation indicating the spread of the force-length curve was set to 0.7 (Challis, 2000).

Various sources have reported that tendon strain under maximum isometric force is 0.04 (e.g., Morgan et al., 1978; Woittiez et al., 1984; Bobbert et al., 1986), although lower values are sometimes used (e.g., 0.03 in Zajac, 1989) and larger values have been reported, for example, for the gastrocnemius (e.g., Arampatzis et al., 2005). In vivo assessment of the tendon strain under maximum isometric force for the FDI produces values around 0.04 (Cook and McDonagh, 1996; Infantolino and Challis, 2014). Therefore the simulation results are presented for a peak tendon strain value of 0.04 , although other feasible tendon strains produced the same pattern of results.

The maximum isometric force $\left(F_{M A X}\right)$ was determined by multiplying each muscles physiological cross-sectional area by the specific tension of muscle $\left(41 \mathrm{~N} / \mathrm{cm}^{2}\right.$; Marx et 
al., 2006). Errors in the assumed specific tension would be systematic and not influence the evaluations of the muscle model that follow.

Overall, this gives seven model parameters: the moment arm of the muscle $\left(r_{M}\right)$, two related to the tendon $\left(L_{T R}, c\right)$, and four related to the muscle fibers $\left(\theta, F_{\max }, L_{F}, w\right)$. Of these seven parameters five were experimentally determined, the two remaining parameters $(c, w)$ were taken from the literature.

Simulations: Simulations were run to predict the maximum isometric abduction moments produced by the FDI about the second metacarpalphalangeal joint for the typical in vivo range of motion of 20 degrees (Kendall et al., 1993). Ten sets of muscle parameters were used to assess the sensitivity of the model to its parameters: the first eight were the parameters for each of the eight muscles, the ninth the mean of the eight parameters sets (Mean 1), and the tenth the mean of three of the original eight muscles (Mean 2). In the often used data set of Wickiewicz et al. (1983) the data were obtained from three cadavers, analysis of their data for optimum fiber length gave a coefficient of variation of $16 \%$. For the tenth data set, Mean 2, a sub-set of three samples from the full data set of eight cadavers analyzed was selected which gave an equivalent coefficient of variation to Wickiewicz et al. (1983) for optimum fiber length.

Results: The sample of muscles provided a range of muscle model parameters (Table 4). For each muscle simulations were run to predict the maximum isometric abduction moments produced by the FDI, the variation in individual muscle properties meant each muscle produced different strength curves (Figure 3). Comparisons were made if the properties for muscle 1 were used to predict the activity of muscle 2 , and so on for each possible combination (Table 5). Generally the percent root mean square differences between the actual muscles joint moments and those predicted using parameters from the other muscles were poor, even when means of the data set were used.

$<<<$ Insert Figure 3, Tables $4 \& 5$ around here. $>>$ 


\section{DISCUSSION}

It is becoming increasingly easier to formulate models of the human musculoskeletal system in part because of increased computer power (Golio, 2015), and because of the development of software environments specifically designed for musculoskeletal modelling (e.g., Davoodi and Loeb, 2002; Delp et al., 2007). While musculoskeletal models can be formulated and verified, there is still the problem of determining the parameters required for the model. Once an appropriate set of model parameters have been identified validation is still problematic, "The basic dilemma in the process of validation may be stated in the following manner: a mathematical analogue can be validated only in a given number of known situations. Yet the main purpose of an analogue is to predict behavior in unknown situations. Thus, no perfect validation is possible." (Panjabi, 1979; page 238). The hope is that while perfect validation is not feasible the model will provide empirical adequacy. Cook and Robinson (2016) have provided evidence of the problems of identifying the parameters for models with nonlinear components, and therefore problems with their validation. In this study two different models were used to examine how the models responded to different data sets. The results highlight two things, that two different models are both sensitive to their model parameters, and that this sensitivity makes the criteria for model validation hard to identify.

For Model 1, examining jumping performance, the discrepancy between the performance of the mean parameters model and the mean performance of the 15 subject specific models, reinforces the conclusions of Cook and Robertson (2016). It is not failings in the model representation but the fundamental non-linear relationships between model inputs and outputs that have limited the simulation accuracy. Even if the simulation based on group mean model parameters (Average 1) is considered relatively close to the mean group performance (falling within a standard deviation), it still does not give a set of performance results (reactivity coefficient, jump height, and time of ground contact) representative of any of the 15 subject specific models. 
For Model 1, model parameters were also determined based on the mean strength and anthropometry of the subjects (Average 2). This gave a model that performed even less like the mean performance of the 15 subject specific models, buts its performance was the closest to subject 8 . There exists non-linear relationships between the data on which the models parameters are determined and the model parameters, and between the model parameters and model output. Both of these nonlinearities highlight the shortcomings of using model parameters based on group data to imply individual or group performance.

For Model 2, simulation of FDI action, cadaver-specific outputs were compared to the model outputs from parameter sets of the average of the cadavers, and the average of three cadavers that best represented the variance in the cadavers of Wickiewicz et al. (1983). These simulations did not produce accurate representations of any of the individual cadaver data sets when mean data sets were used, and that individual data sets do not approximate well to other individual data sets. The differences in moment production has implications for the use of cadaveric musculoskeletal parameters in musculoskeletal models as many models use one or more cadaveric sources as inputs to predict live subject motion. There are muscle model parameters sets which can be used for muscle modelling which are based on mean data (e.g., Ward et al., 2009), and those based on single cadaver analysis (Klein Horsman et al., 2007). The analysis from Model 2 suggest that models parameters need to be subject specific to accurately reflect the moments that a subject may produce.

Studies using models vary in their purpose, they may be examining the performance of a specific subject (e.g., Allen et al., 2011), the overall performance of a group of subjects (e.g., Jacobs et al., 1996), or examining general principles of human movement (e.g., Alexander, 1990). Given the different purposes of such models the level of accuracy of validation can vary, with for example subject specific modelling requires higher accuracy than a study examining general principles. The present study has provided evidence that averaging data to determine model parameters can be an 
inappropriate approach, but its appropriateness should be considered in light of the source of the model parameters and the purpose of the model.

One potential critique of this study is that the models were not robust with respect to their parameters. The model of jumping has been examined previously to determine it sensitivity to the model parameters (Alexander, 1990; Challis, 1998). Both of these studies have demonstrated that the model is relatively insensitive to small errors in its model parameters. The model of the FDI muscle is an example of Hill-type muscle model. Out et al. (1996) examined the sensitivity of a Hill-type muscle model to its parameters, and found it was robust to all parameters except tendon resting length, and muscle maximum isometric force. Scovil and Ronsky (2006) reached the same conclusion but also identified the muscle force-length properties. For Model 2 measures were made directly on cadavers muscles to allow determination of these three identified model parameters; for each cadaver specimen there was a unique set of these parameters.

Ockham's Razor is often invoked to justify selecting a relatively simple model, which comes with the advantage that the model parameters are few and therefore in theory easy to determine. Of course simply invoking Ockham's Razor does not justify a model's level of complexity, but complex models do create problems with the identification of model parameters and the sensitivity of the model to its parameters. In this study it has been shown for a simple model of jumping (cf. Anderson and Pandy, 2001), and a relatively simple model of a muscle (cf. Ma and Zahalak, 1991) that the models are very sensitive to their parameters. Should more complex models have been used, the greater presence of non-linearities in the models would have ensured a similar, if not greater, sensitivity to the model parameters (Cook and Robertson, 2017).

It has previously been highlighted how well math can be used to examine phenomena in the natural sciences (e.g., Wigner, 1960). The problem with models is that given sufficient model parameters good fits can almost be ensured of the identified system (Dyson, 2004). To ensure the model is appropriate two things should be established for 
the model: its internal, and external validity. Internal validity is the appropriate representation of the components of the model, for example the representation of the muscle fiber force-length properties in the model. External validity is the comparison of the model performance with actual performance. A model may have external validity, and have some utility as a consequence, but if it does not have internal validity it may not reveal much about the internal working of the system under evaluation. The focus of the present study was model parameters not model appropriateness per se, but to test the appropriateness of a model a suitable set of model parameters are a necessity, this study has highlighted some of the issues associated with identifying a set of model parameters.

This study has examined the sensitivity of two different musculoskeletal models to the parameters describing the model. These two different models were both sensitive to the model parameters which help define them. The results suggest that the practise of using mean group data to parameterize a model is inappropriate. Finally, the criteria for model validation was also addressed, where using mean group data did not provide data reflective of any of the population from which the model parameters was based. The cumulative influence of this study is that musculoskeletal models should ideally have customized model parameters, and carefully justify their validation rather than use a general yet potentially inappropriate criteria.

\section{DISCLOSURE STATEMENT}

No potential conflict of interest was reported by the authors. 


\section{REFERENCES}

Alexander, R. M. 1990. Optimum take-off techniques for high and long jumps. Philosophical Transactions of the Royal Society, Series B, 329, 3-10.

Allen, S. J., King, M. A., Yeadon, M. R. 2011. Is a single or double arm technique more advantageous in triple jumping? Journal of Biomechanics, 43(16), 3156-3161.

An, K. N., Ueba, Y., Chao, E. Y., Cooney, W. P., Linscheid, R. L. 1983. Tendon excursion and moment arm of index finger muscles. Journal of Biomechanics, 16(6), 419-425.

Anderson, F. C., Pandy, M. G. 2001. Dynamic optimization of human walking. Journal of Biomechanical Engineering, 123, 381-390.

Arampatzis, A., Stafilidis, S., DeMonte, G., Karamanidis, K., Morey-Klapsing, G., Bruggemann, G. P. 2005. Strain and elongation of the human gastrocnemius tendon and aponeurosis during maximal plantarflexion effort. Journal of Biomechanics, 38(4), 833-841.

Baskin, R. J., Paolini, P. J. 1966. Muscle volume changes. Journal of General Physiology, 49(3), 387-404.

Bobbert, M. F., Brand, C., de Hann, A., Huijing, P. A., van Ingen Schenau, G. J., Rijnsburger, W. H., Woittiez, R. D. 1986. Series elasticity of tendinous structures of the the rat EDL. Journal of Physiology, 377, 89P.

Challis, J. H. 1998. An investigation of the influence of bi-lateral deficit on human jumping. Human Movement Science, 17(3), 307-325.

Challis, J. H. 2000. Muscle-tendon architecture and athletic performance. In V. M. Zatsiorsky (Ed.), Biomechanics in Sport. (Olympic Encyclopaedia of Sports Medicine Vol. IX) (pp. 33-55). Oxford: Blackwell Science Ltd.

Cheng, K. B., Hubbard, M. 2004. Optimal jumping strategies from compliant surfaces: A simple model of springboard standing jumps. Human Movement Science, 23(1), 35-48.

Close, R. 1964. Dynamic properties of fast and slow skeletal muscles of the rat during development. Journal of Physiology, 173, 74-95.

Cook, C. S., McDonagh, M. J. 1996. Measurement of muscle and tendon stiffness in man. European Journal of Applied Physiology, 72(4), 380-382.

Cook, D. D., Robertson, D. J. 2016. The generic modeling fallacy: Average biomechanical models often produce non-average results!. Journal of Biomechanics, 49(15), 3609-3615.

Corana, A., Marchesi, M., Martini, C., Ridella, S. 1987. Minimizing multimodal functions of continuous variables with the "simulated annealing" algorithm. ACM Transactions on Mathematical Software, 13(3), 262-280.

Cronin, J. B., Hansen, K. T. 2005. Strength and power predictors of sports speed. Journal of Strength and Conditioning Research, 19(2), 349-357.

Davoodi, R., Loeb, G. E. 2002. A software tool for faster development of complex models of the musculoskeletal systems and sensorimotor controllers in Simulink. Journal of Applied Biomechanics, 18(4), 357-365.

Delp, S. L., Anderson, F. C., Arnold, A. S., Loan, P., Habib, A., John, C. T., Guendelman, E., Thelen, D. G. 2007. OpenSim: Open-source software to create and analyze dynamic simulations of movement. IEEE Transactions on Biomedical Engineering, 54(11), 1940-1950. 
Delp, S. L., Loan, J. P., Hoy, M. G., Zajac, F. E., Topp, E. L., Rosen, J. M. 1990. An interactive graphics-based model of the lower extremity to study orthopaedic surgical procedures. IEEE Transactions on Biomedical Engineering, 37(8), 757767.

Dorn, T. W., Schache, A. G., Pandy, M. G. 2012. Muscular strategy shift in human running: dependence of running speed on hip and ankle muscle performance. The Journal of Experimental Biology, 215(11), 1944-1956.

Dyson, F. (2004). A meeting with Enrico Fermi. Nature, 427(6972), 297.

Fetzer, J. H. 1988. Program verification - the very idea. Communications of the ACM, 31(9), 1048-1063.

Forrester, S. E., Yeadon, M. R., King, M. A., Pain, M. T. 2011. Comparing different approaches for determining joint torque parameters from isovelocity dynamometer measurements. Journal of Biomechanics, 44(5), 955-961.

Fox, M. D., Reinbolt, J. A., Ounpuu, S., Delp, S. L. 2009. Mechanisms of improved knee flexion after rectus femoris transfer surgery. Journal of Biomechanics, 42(5), 614619.

Friederich, J. A., Brand, R. A. 1990. Muscle fiber architecture in the human lower limb. Journal of Biomechanics, 23(1), 91-95.

Golio, M. 2015. Fifty years of Moore's law. Proceedings of the IEEE, 103(10), 19321937.

Gordon, A. M., Huxley, A. F., Julian, F. J. 1966. The variation in isometric tension with sarcomere length in vertebrate muscle fibres. Journal of Physiology, 184(1), 170192.

Hill, A. V. 1938. The heat of shortening and dynamic constants of muscle. Proceedings of the Royal Society, Series B, 126, 136-195.

Hoy, M. G., Zajac, F. E., Gordon, M. E. 1990. A musculoskeletal model of the human lower extremity: The effect of muscle, tendon, and moment arm on the momentangle relationship of musculotendon actuators at the hip, knee, and ankle. Journal of Biomechanics, 23(2), 157-169.

Huijing, P. A. 1985. Architecture of the human gastrocnemius muscle and some functional consequences. Acta Anatomica, 123(2), 101-107.

Infantolino, B. W., Challis, J. H. 2010. Architectural properties of the first dorsal interosseous. Journal of Anatomy, 216(4), 463-469.

Infantolino, B. W., Challis, J. H. 2014. Measuring subject specific muscle model parameters of the First Dorsal Interosseous in vivo. Annals of Biomedical Engineering, 42(6), 1331-1339.

Jacobs, R., Bobbert, M. F., van Ingen Schenau, G. J. 1996. Mechanical output from individual muscles during explosive leg extensions: the role of biarticular muscles. Journal of Biomechanics, 29(4), 513-523.

Karamanidis, K., Arampatzis, A. 2006. Mechanical and morphological properties of human quadriceps femoris and triceps surae muscle-tendon unit in relation to aging and running. Journal of Biomechanics, 39(3), 406-417.

Kendall, F. P., McCreary, E. K., Provance, P. G. 1993. Muscles, Testing and Functions. Baltimore: Lippincott Williams \& Wilkins.

Klein Horsman, M. D., Koopman, H. F. J. M., van der Helm, F. C. T., Prose, L. P., Veeger, H. E. J. 2007. Morphological muscle and joint parameters for 
musculoskeletal modelling of the lower extremity. Clinical Biomechanics, 22(2), 239-247.

Lichtwark, G. A., Wilson, A. M. 2008. Optimal muscle fascicle length and tendon stiffness for maximising gastrocnemius efficiency during human walking and running. Journal of Theoretical Biology, 252(4), 662-673.

Ma, S., Zahalak, G. I. 1991. A distribution-moment model of energetics in skeletal muscle. Journal of Biomechanics, 24(1), 21-35

Marx, J. O., Olsson, M. C., Larsson, L. 2006. Scaling of skeletal muscle shortening velocity in mammals representing a 100,000-fold difference in body size. Pflugers Archiv-European Journal of Physiology, 452, 222-230.

Masquelet, A. C., Salama, J., Outrequin, G., Serrault, M., Chevrel, J. P. 1986. Morphology and functional anatomy of the first dorsal interosseous muscle of the hand. Surgical and Radiologic Anatomy, 8, 19-28.

Ma, S., Zahalak, G. I. 1991. A distribution-moment model of energetics in skeletal muscle. Journal of Biomechanics, 24(1), 21-35.

McErlain-Naylor. S, King, M.A., Pain, M.T.G., 2014. Determinants of countermovement jump performance: a kinetic and kinematic analysis. Journal of Sports Sciences, 32(19), 1805-1812.

Miller, R. H., Hamill, J. 2009. Computer simulation of the effects of shoe cushioning on internal and external loading during running impacts. Computer Methods in Biomechanics and Biomedical Engineering, 12(4), 481-490.

Morgan, D. L., Proske, U., Warren, D. 1978. Measurement of muscle stiffness and the mechanism of elastic storage in hopping kangaroos. Journal of Physiology, 282, 253-261.

Narici, M. 1999. Human skeletal muscle architecture studied in vivo by non-invasive imaging techniques: functional significance and applications. Journal of Electromyography and Kinesiology, 9(2), 97-103.

Neptune, R. R., Kautz, S. A., Zajac, F. E. 2000. Muscle contributions to specific biomechanical functions do not change in forward versus backward pedaling. Journal of Biomechanics, 323(2), 155-164.

Otten, E. 1988. Concepts and models of functional architecture in skeletal muscle. Exercise and Sport Sciences Reviews, 16, 89-137.

Out, L., Vrijkotte, T. G. M., van Soest, A. J., \& Bobbert, M. F. (1996). Influence of the parameters of a human triceps surae muscle model on the isometric torqueangle relationship. Journal of Biomechanical Engineering, 118(1), 17-25.

Pain, M.T.G., Young, F., Kim J.W., Forrester, S.E., 2013. The torque-velocity relationship in large human muscles: maximum voluntary versus electrically stimulated behaviour. Journal of Biomechanics, 46, 645-650.

Pain, M.T.G., 2014. Considerations for single and double leg drop jumps: Bilateral deficit, standardising drop height and equalising training load. Journal of Applied Biomechanics, 30(6), 722-727.

Panjabi, M. 1979. Validation of mathematical models. Journal of Biomechanics, 12(3), 238.

Pierrynowski, M. R. 1995. Analytic representation of muscle line of action and geometry. In P. Allard, I. A. F. Stokes and J. P. Blanchi (Eds.), Three-Dimensional Analysis 
of Human Movement (pp. 215-256). Champaign, Illinois: Human Kinetics Publishers.

Schwer, L. E. 2002. ASME Standards Committee on verification and validation in computational solid mechanics ASME Council on Codes and Standards.

Scovil, C. Y., \& Ronsky, J. L. (2006). Sensitivity of a Hill-based muscle model to perturbations in model parameters. Journal of Biomechanics, 39(11), 2055-2063.

Seyfarth, A., Blickhan, R., van Leeuwen, J. L. 2000. Optimum take-off techniques and muscle design for long jump. Journal of Experimental Biology, 203(4), 741-750.

Slager, G. E., Otten, E., van Eijden, T. M., van Willigen, J. D. 1997. Mathematical model of the human jaw system simulating static biting and movements after unloading. Journal of Neurophysiology, 78(6), 3222-3233.

Valero-Cuevas, F. J., Hoffmann, H., Kurse, M. U., Kutch, J. J., Theodorou, E. A. 2009. Computational models for neuromuscular function. IEEE Reviews in Biomedical Engineering, 2, 110-135.

Van Soest, A. J., Schwab, A. L., Bobbert, M. F., Van Ingen Schenau, G. J. 1993. The influence of the biarticularity of the gastrocnemius muscle on vertical-jumping achievement. Journal of Biomechanics, 26(1), 1-8.

Walker, S. M., Schrodt, G. R. 1973. I segment lengths and thin filament periods in skeletal muscle fibers of the rhesus monkey and the human. Anatomical Record, 178, 63-82.

Ward, S. R., Eng, C. M., Smallwood, L. H., Lieber, R. L. 2009. Are current measurements of lower extremity muscle architecture accurate? Clinical Orthopaedics and Related Research, 467(4), 1074-1082.

Westing, S. H., Cresswell, A. G., Thorstensson, A. 1991. Muscle activation during maximal voluntary eccentric and concentric knee extension. European Journal of Applied Physiology and Occupational Physiology, 62(2), 104-108.

Wickiewicz, T. L., Roy, R. R., Powell, P. L., Edgerton, V. R. 1983. Muscle architecture of the human lower limb. Clinical Orthopaedics and Related Research, 179, 275283.

Wigner, E. P. 1960. The unreasonable effectiveness of mathematics in the natural sciences. Communications on Pure and Applied Mathematics, 13(1), 1-14.

Woittiez, R. D., Huijing, P. A., Boom, H. B. K., Rozendal, R. H. 1984. A threedimensional muscle model: A quantified relation between form and function of skeletal muscle. Journal of Morphology, 182(1), 95-113.

Yeadon, M. R., King, M. A., Wilson, C. 2006. Modelling the maximum voluntary joint torque/angular velocity relationship in human movement. Journal of Biomechanics, 39(3), 476-482.

Zajac, F. E. 1989. Muscle and tendon: properties, models, scaling, and application to biomechanics and motor control. CRC Critical Reviews in Biomedical Engineering, 17(4), 359-411. 


\section{LIST OF TABLES}

Table 1: The anthropometric details of the fifteen subjects, and the group means $( \pm$ standard deviation).

Table 2: The knee extensor model parameters for the fifteen subjects, and the group means ( \pm standard deviation).

Table 3: Reactivity coefficient (RC), jump height (JH), and time of ground contact (tGc) for the fifteen subject specific models, along with the mean parameter results, and the simulations based on mean group data. The first mean data set, Average 1, was the mean from the subject specific model parameters, while Average 2 was determined from the mean measured human strength and anthropometric data.

Table 4: Each of the cadavers' basic anthropometry and muscle specific parameters.

Table 5: Percent root mean square differences between the model outputs of joint moments for eight cadaver-specific muscles, the average of the muscles (Mean 1), and the Wickiewicz et al. average (Mean 2). Moments were produced during isometric simulations throughout a 20 degree range of motion. 


\section{TABLES}

Table 1: The anthropometric details of the 15 subjects, and the group means ( \pm standard deviation).

\begin{tabular}{ccccc}
\hline $\begin{array}{c}\text { Subject } \\
\#\end{array}$ & $\begin{array}{c}\text { Body Mass } \\
(\mathbf{k g})\end{array}$ & $\begin{array}{c}\text { Height } \\
(\mathbf{m})\end{array}$ & $\begin{array}{c}\text { Shank Length } \\
(\mathbf{m m})\end{array}$ & $\begin{array}{c}\text { Thigh Length } \\
(\mathbf{m})\end{array}$ \\
\hline 1 & 75.4 & 1.71 & 405 & 415 \\
\hline 2 & 102.8 & 1.89 & 480 & 465 \\
\hline 3 & 76.0 & 1.76 & 430 & 435 \\
\hline 4 & 81.6 & 1.75 & 420 & 435 \\
\hline 5 & 75.6 & 1.75 & 415 & 410 \\
\hline 6 & 91.0 & 1.75 & 410 & 430 \\
\hline 7 & 68.6 & 1.79 & 425 & 420 \\
\hline 8 & 73.5 & 1.75 & 415 & 395 \\
\hline 9 & 56.1 & 1.69 & 367 & 365 \\
\hline 10 & 85.0 & 1.87 & 465 & 474 \\
\hline 11 & 75.2 & 1.76 & 409 & 437 \\
\hline 12 & 66.0 & 1.78 & 401 & 415 \\
\hline 13 & 74.8 & 1.83 & 422 & 449 \\
\hline 14 & 73.9 & 1.77 & 387 & 367 \\
\hline 15 & 68.7 & 1.80 & 403 & 402 \\
\hline Mean & $76.3 \pm 10.9$ & $1.78 \pm 0.05$ & $417 \pm 28$ & $421 \pm 31$ \\
\hline
\end{tabular}


Table 2: The knee extensor model parameters for the 15 subjects, and the group means ( \pm standard deviation).

\begin{tabular}{|c|c|c|c|c|c|c|c|c|}
\hline $\begin{array}{c}\text { Subject } \\
\#\end{array}$ & $\begin{array}{c}T_{E C C} \\
(\mathrm{Nm}) \\
\end{array}$ & $\begin{array}{c}T_{o} \\
(\mathrm{Nm})\end{array}$ & $\begin{array}{c}\omega_{\text {MAX }} \\
\left(\operatorname{rad~s}^{-1}\right)\end{array}$ & $\begin{array}{c}\omega_{c} \\
\left(\mathrm{rad} \mathrm{s}^{-1}\right)\end{array}$ & $\begin{array}{c}a_{\text {MIN }} \\
(-)\end{array}$ & $\begin{array}{c}\omega_{r} \\
\left(\mathrm{rad} \mathrm{s}^{-1}\right)\end{array}$ & $\begin{array}{c}\omega_{1} \\
\left(\mathrm{rad} \mathrm{s}^{-1}\right)\end{array}$ & $\begin{array}{c}k_{t} \\
\left(\mathrm{Nm} \mathrm{rad}^{-1}\right)\end{array}$ \\
\hline 1 & 465 & 312 & 20.8 & 6.3 & 0.768 & 0.929 & -0.035 & 778 \\
\hline 2 & 526 & 375 & 18.0 & 5.4 & 0.894 & 0.471 & -0.701 & 1040 \\
\hline 3 & 507 & 361 & 23.9 & 8.4 & 0.647 & 0.923 & 0.768 & 737 \\
\hline 4 & 461 & 279 & 21.8 & 6.5 & 0.735 & 0.936 & -0.303 & 702 \\
\hline 5 & 308 & 197 & 19.6 & 5.9 & 0.903 & 0.497 & -1.307 & 533 \\
\hline 6 & 480 & 303 & 19.9 & 6.5 & 0.842 & 0.654 & -0.911 & 876 \\
\hline 7 & 485 & 309 & 18.3 & 5.5 & 0.653 & 1.563 & 1.475 & 582 \\
\hline 8 & 521 & 345 & 22.3 & 7.1 & 0.688 & 1.153 & 0.560 & 748 \\
\hline 9 & 248 & 141 & 27.7 & 8.3 & 0.836 & 0.894 & -1.402 & 340 \\
\hline 10 & 467 & 323 & 27.7 & 10.7 & 0.807 & 0.431 & 0.918 & 700 \\
\hline 11 & 400 & 230 & 32.0 & 13.1 & 0.761 & 1.571 & -1.073 & 572 \\
\hline 12 & 406 & 260 & 19.3 & 15.8 & 0.752 & 0.468 & -0.389 & 596 \\
\hline 13 & 378 & 251 & 35.6 & 10.7 & 0.879 & 0.717 & -1.468 & 626 \\
\hline 14 & 326 & 208 & 29.7 & 9.4 & 0.863 & 0.834 & -1.477 & 539 \\
\hline 15 & 332 & 185 & 18.1 & 14.7 & 0.854 & 0.102 & -0.184 & 451 \\
\hline Mean & $\begin{array}{l}421 \\
\pm 86\end{array}$ & $\begin{array}{l}272 \\
\pm 69 \\
\end{array}$ & $\begin{array}{l}23.6 \\
\pm 5.6 \\
\end{array}$ & $\begin{array}{c}8.95 \\
\pm 3.39 \\
\end{array}$ & $\begin{array}{c}0.792 \\
\pm 0.085 \\
\end{array}$ & $\begin{array}{c}0.810 \\
\pm 0.407\end{array}$ & $\begin{array}{l}-0.369 \\
\pm 0.950 \\
\end{array}$ & $\begin{array}{c}655 \\
\pm 173 \\
\end{array}$ \\
\hline
\end{tabular}

Note: $T_{E C C}$ - maximum moment produced under eccentric conditions (per leg), $T_{O}$ maximum isometric moment (per leg), $\omega_{M A X}$ - maximum concentric angular velocity, $\omega_{c}-$ constant, $a_{M I N}$ - lowest level of activation in eccentric phase, $\omega_{r}$-governs the rate at which activation increases with angular velocity, $\omega_{1}$ - the angular velocity at the midpoint in activation rise, and $k_{t}$ - tendon stiffness. 
Table 3: Reactivity coefficient (RC), jump height (JH), and time of ground contact (tGc) for the fifteen subject specific models, along with the mean parameter results, and the simulations based on mean group data. The first mean data set, Mean 1, was the mean from the subject specific model population data, while Mean 2 was determined from the mean measured human strength and anthropometric data.

\begin{tabular}{cccc}
\hline Subject \# & $\begin{array}{c}\mathbf{R C} \\
\left(\mathbf{m ~ s}^{-1}\right)\end{array}$ & $\begin{array}{c}\mathbf{J H} \\
(\mathbf{c m})\end{array}$ & $\begin{array}{c}\mathbf{t G C} \\
(\mathbf{m s})\end{array}$ \\
\hline 1 & 1.81 & 17.3 & 95 \\
\hline 2 & 1.28 & 15.2 & 118 \\
\hline 3 & 1.54 & 18.6 & 121 \\
\hline 4 & 1.04 & 14.0 & 134 \\
\hline 5 & 0.67 & 11.0 & 164 \\
\hline 6 & 1.26 & 14.2 & 113 \\
\hline 7 & 1.86 & 19.2 & 103 \\
\hline 8 & 2.18 & 19.5 & 89 \\
\hline 9 & 0.89 & 13.1 & 148 \\
\hline 10 & 0.84 & 19.3 & 231 \\
\hline 11 & 0.73 & 16.5 & 226 \\
\hline 12 & 1.77 & 18.5 & 105 \\
\hline 13 & 1.00 & 18.4 & 185 \\
\hline 14 & 1.00 & 14.0 & 139 \\
\hline 15 & 0.96 & 13.4 & 140 \\
\hline Mean Performance & $1.25 \pm 0.47$ & $16.1 \pm 2.7$ & $141 \pm 44$ \\
\hline Simulated: Mean 1 & 1.39 & 16.6 & 120 \\
\hline Simulated: Mean 2 & 2.10 & 19.5 & 93 \\
\hline & & & \\
\hline & & & 140 \\
\hline
\end{tabular}


Table 4: For each of the cadaver basic anthropometry and muscle specific parameters.

\begin{tabular}{cccccccc}
\hline $\begin{array}{c}\text { Muscle } \\
\#\end{array}$ & $\begin{array}{c}\text { Height } \\
(\mathbf{c m})\end{array}$ & $\begin{array}{c}\text { Metacarpal } \\
\text { Two } \\
\text { Length } \\
(\mathbf{m m})\end{array}$ & $\begin{array}{c}\text { Optimum } \\
\text { Fiber } \\
\text { Length } \\
(\mathbf{m m})\end{array}$ & $\begin{array}{c}\text { Tendon } \\
\text { Length } \\
(\mathbf{m m})\end{array}$ & $\begin{array}{c}\text { PCSA } \\
\left(\mathbf{c m}^{2}\right)\end{array}$ & $\begin{array}{c}\text { Pennation } \\
\text { Angle } \\
(\text { Degree })\end{array}$ & $\begin{array}{c}\text { Moment } \\
\text { Arm } \\
(\mathbf{c m})\end{array}$ \\
\hline 1 & 161 & 62.4 & 22.15 & 3.0 & 1.67 & 22.5 & 0.18 \\
\hline 2 & 161 & 55.1 & 33.14 & 2.0 & 0.85 & 20 & 0.26 \\
\hline 3 & 180 & 55.8 & 29.95 & 5.0 & 2.01 & 17.5 & 0.22 \\
\hline 4 & 180 & 48.6 & 31.93 & 7.0 & 1.82 & 14.5 & 0.25 \\
\hline 5 & 174 & 68.1 & 24.76 & 0.0 & 2.61 & 15.5 & 0.19 \\
\hline 6 & 174 & 48.4 & 61.04 & 4.0 & 0.93 & 19.5 & 0.16 \\
\hline 7 & 169 & 51.0 & 33.84 & 2.0 & 2.25 & 17.5 & 0.23 \\
\hline 8 & 169 & 45.4 & 28.11 & 2.0 & 2.63 & 22.5 & 0.19 \\
\hline Mean & 171 & 54.3 & 33.12 & 3.1 & 1.85 & 18.7 & 0.21 \\
\hline SD & 7.4 & 7.7 & 12.0 & 2.2 & 0.68 & 3.0 & 0.04 \\
\hline
\end{tabular}


Table 5: Percent root mean square differences between the model outputs of joint moments for eight cadaver-specific muscles, the average of the muscles (Mean 1), and the Wickiewicz et al. average (Mean 2). Moments were produced during isometric simulations throughout a 20 degree range of motion.

\begin{tabular}{ccccccccccc}
\hline $\begin{array}{c}\text { Muscle } \\
\#\end{array}$ & $\mathbf{1}$ & $\mathbf{2}$ & $\mathbf{3}$ & $\mathbf{4}$ & $\mathbf{5}$ & $\mathbf{6}$ & $\mathbf{7}$ & $\mathbf{8}$ & Mean 1 & Mean 2 \\
\hline $\mathbf{1}$ & $\mathbf{-}$ & 46.3 & 87.8 & 77.8 & 120.5 & 77.7 & 92.9 & 104.4 & 31.3 & 101.8 \\
\hline $\mathbf{2}$ & 84.8 & - & 245.5 & 227.0 & 305.5 & 59.2 & 254.4 & 276.0 & 141.9 & 271.1 \\
\hline $\mathbf{3}$ & 46.8 & 71.5 & - & 6.0 & 17.6 & 88.1 & 6.3 & 8.9 & 30.2 & 7.6 \\
\hline $\mathbf{4}$ & 43.8 & 69.7 & 6.3 & - & 24.7 & 87.5 & 9.0 & 15.2 & 26.2 & 13.6 \\
\hline $\mathbf{5}$ & 54.8 & 75.9 & 15.0 & 20.0 & - & 89.9 & 14.8 & 7.8 & 40.7 & 9.3 \\
\hline $\mathbf{6}$ & 347.4 & 144.5 & 739.1 & 695.2 & 884.3 & - & 763.0 & 813.7 & 487.4 & 802.1 \\
\hline $\mathbf{7}$ & 48.2 & 72.0 & 6.2 & 8.3 & 16.9 & 88.4 & - & 8.2 & 32.0 & 6.5 \\
\hline $\mathbf{8}$ & 51.1 & 73.8 & 8.2 & 13.3 & 8.4 & 89.1 & 7.7 & - & 35.9 & 1.6 \\
\hline Mean 1 & 23.8 & 59.0 & 43.2 & 35.4 & 68.2 & 83.0 & 47.0 & 55.8 & - & 53.7 \\
\hline Mean 2 & 50.5 & 73.4 & 7.1 & 12.0 & 10.1 & 89.0 & 6.2 & 1.6 & 35.0 & - \\
\hline
\end{tabular}




\section{LIST OF FIGURES}

Figure 1: A schematic representation of the moment driven jumping model.

Figure 2. Representation of the components of the model of the musculature action of the FDI.

Figure 3: Graph illustrating joint moments for five muscles, including the average muscle, and a Wickiewicz et al. average muscle. The moments were generated during simulations of isometric contractions throughout a 20 degree range of motion and normalized with respect to the maximum moment generated by muscle \#3. 


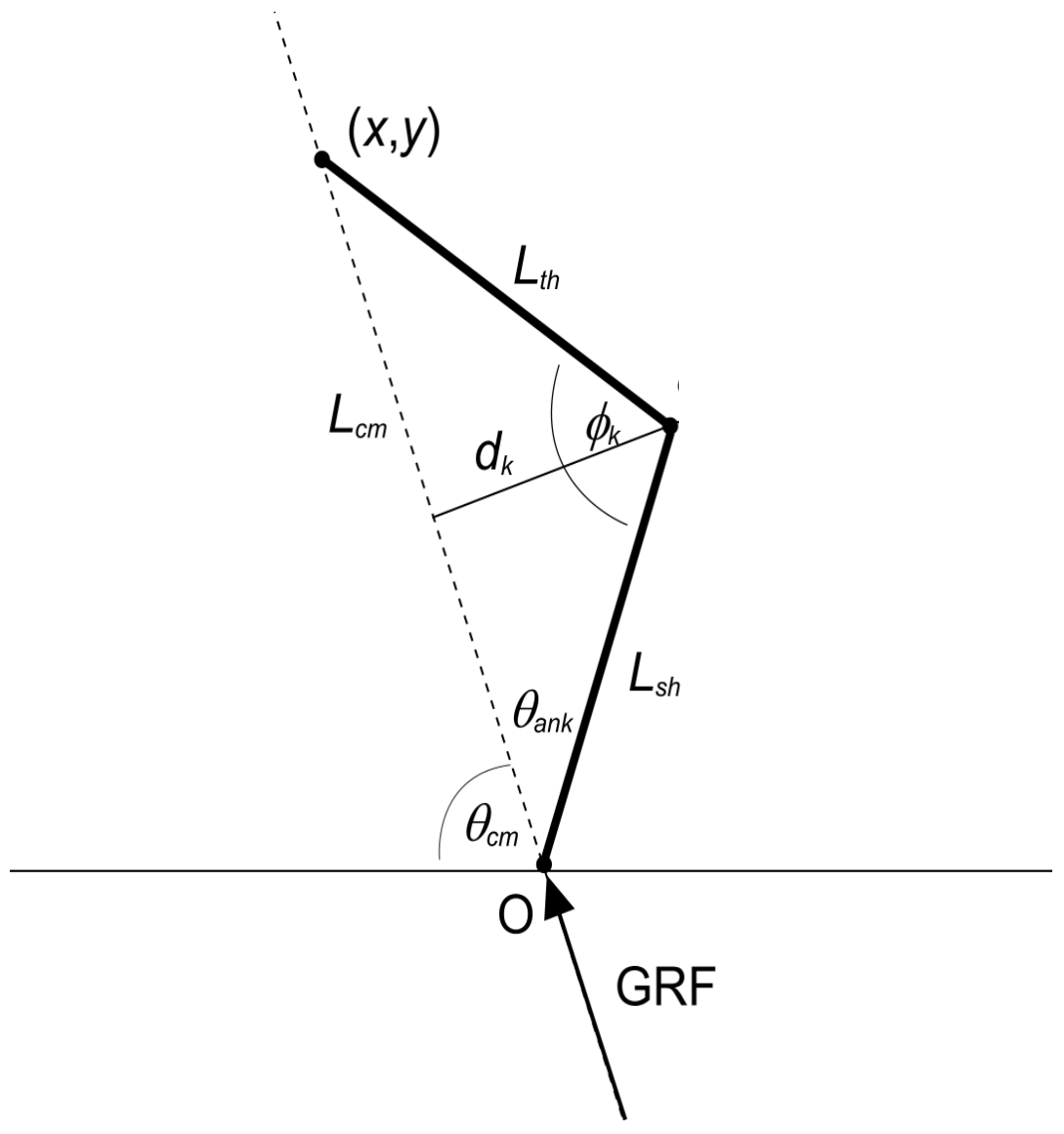

Figure 1: A schematic representation of the torque driven jumping model. 


$$
F_{T}=q \cdot \cos (\theta) \cdot F_{\text {max }} \cdot F_{L}\left(L_{F}\right)
$$
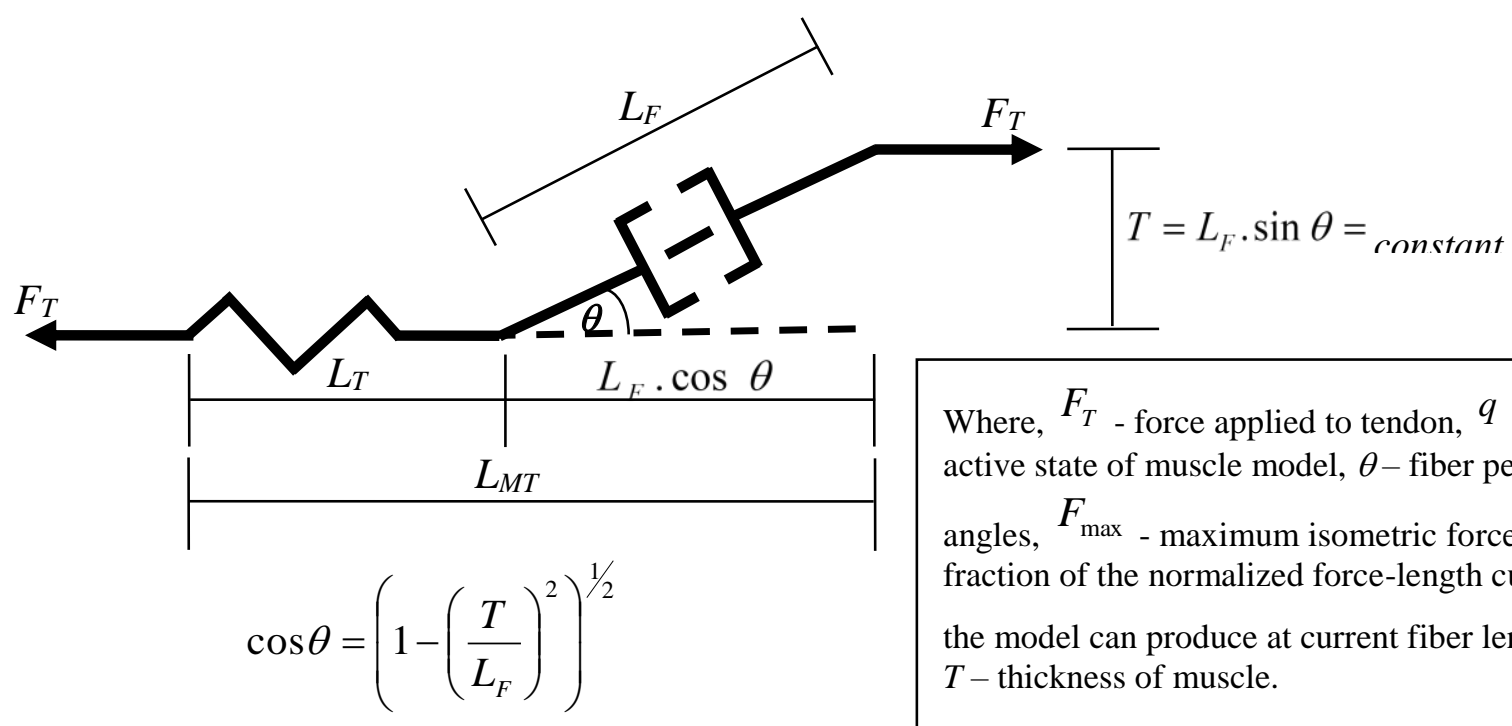

Where, $F_{T}$ - force applied to tendon, ${ }^{q}$ - current active state of muscle model, $\theta$ - fiber pennation angles, $F_{\max }$ - maximum isometric force, $F_{L}\left(L_{F}\right)$. fraction of the normalized force-length curve that the model can produce at current fiber length $\left({ }_{F}\right)$, $T$ - thickness of muscle.

\section{Action of the First Dorsal Interosseous}

$\Delta L_{M T}=r_{m} \cdot \phi \quad M_{J}=F_{T} \cdot r_{m}$

$\Delta L_{M T}$ - change in length of muscle tendon complex

$r_{m}$ - moment arm of the FDI at the second

metacarpalphalangeal joint

$\phi$ - abduction angle of $2^{\text {nd }}$ metacarpalphalangeal joint

$\phi$ - muscle moment at $2^{\text {nd }}$ metacarpalphalangeal joint

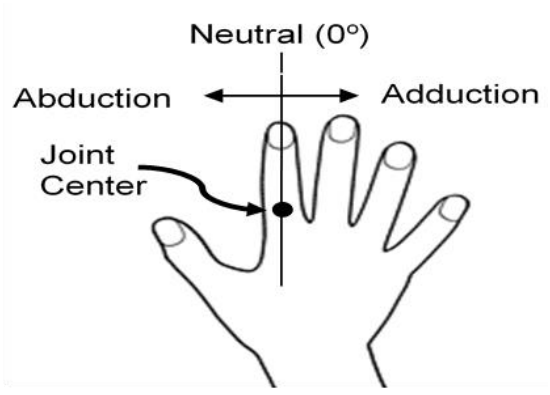

\section{Force-length Properties}

$F_{L}\left(L_{F}\right)=1-\left(\frac{\left(L_{F}-L_{F, O P T}\right)}{w \cdot L_{F, O P T}}\right)^{2}$

$L_{F, O P T}$ - optimum length of muscle fiber

$w$ - parameter indicating width of force-length curve.

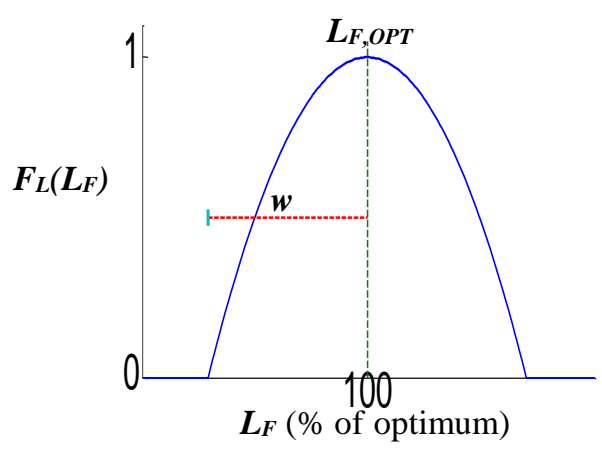

\section{Tendon Properties}

$L_{T}=L_{T R}+\frac{c}{F_{\max }} \cdot L_{T R} \cdot F_{T}$

$L_{T}$ - current length of the tendon

$L_{T R}$ - resting length of the tendon

$c$ - strain in tendon under maximum isometric force

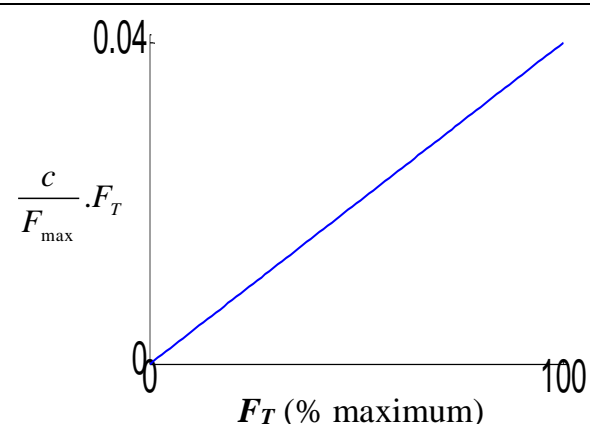

Figure 2. Representation of the components of the model of the musculature action of the FDI. 


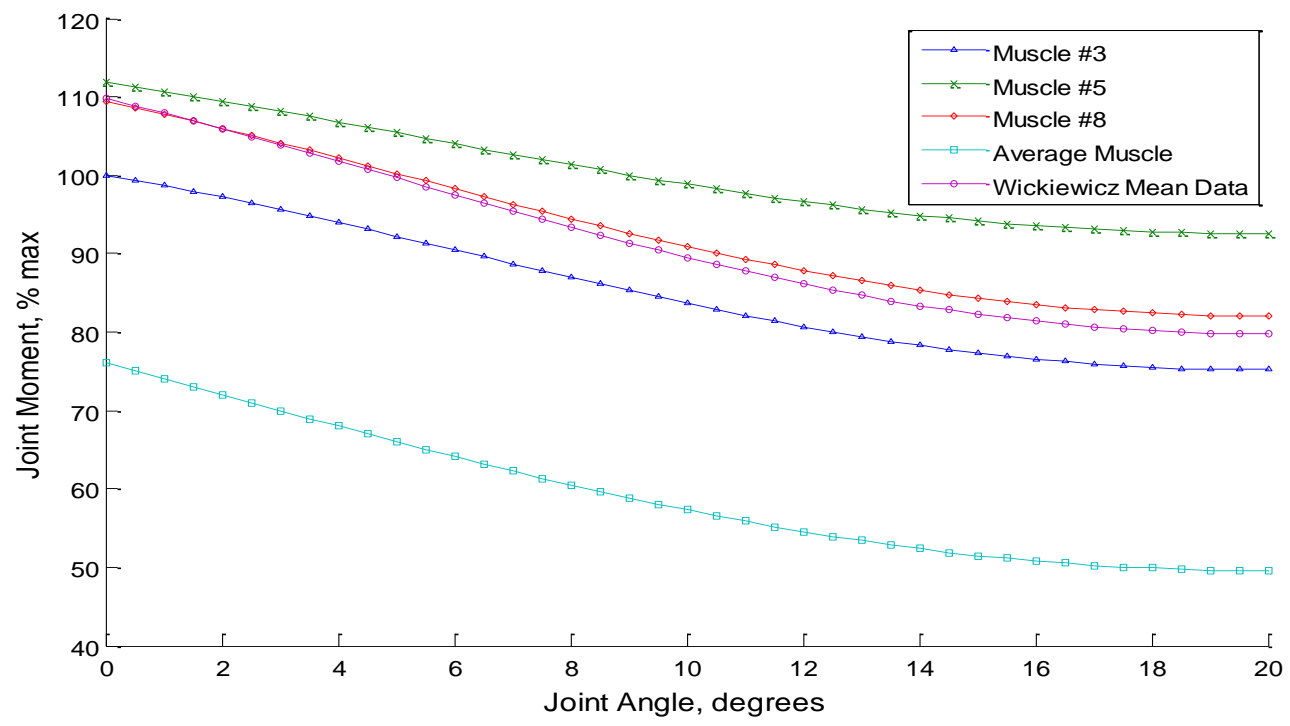

Figure 3: Graph illustrating joint moments for five muscles, including the average muscle, and a Wickiewicz et al. average muscle. The moments were generated during simulations of isometric contractions throughout a 20 degree range of motion and normalized with respect to the maximum moment generated by muscle \#3. 